\title{
Object Identification and Localization using Convolution Neural Network
}

\author{
${ }^{1}$ BontleGoitsemedi and ${ }^{2}$ Kedibonye Keletso \\ 1,2 Electrical and Electronic Engineering, Botswana College of Engineering and Technology. Botswana. \\ ${ }^{1}$ bontlegoit@protonmail.com
}

\begin{abstract}
Article Info
Journal of Computing and Natural Science (http://anapub.co.ke/journals/jens/jens.html)

Doi: https://doi.org/10.53759/181X/JCNS202101006

Received 02 October 2020; Revised form 02 November 2020; Accepted 25 December 2021.

Available online 05 April 2021.

(C2021 Published by AnaPub Publications.
\end{abstract}

\begin{abstract}
Improving object identification against impediment, obscure and clamor image is a basic advance to deploy detector in real time applications. Since it is preposterous to expect to debilitate all picture abandons through information assortment, numerous specialists look to produce hard examples in preparing. The produced hard examples are either pictures or highlight maps with coarse patches exited in the spatial measurements. Huge overheads are needed in preparing the extra hard examples and additionally assessing drop-out patches utilizing additional organization branches. In this paper we proposed GRAD CAM++ with Mask Regional Convolution Neural Network (Mask RCNN) based item limitation and identification. The significant advantages of utilizing Mask R-CNN is that they beat all the partner techniques in the space and can likewise be utilized in unaided environments. The proposed identifier dependent on GRAD CAM++ with Mask R-CNN gives a vigorous and plausible capacity on recognizing and grouping objects exist and their shapes progressively on location. It is discovered that the proposed strategy can perform exceptionally successful and productive in a wide scope of pictures and gives higher goal visual portrayal.
\end{abstract}

Keywords - GRAD CAM++, Mask R-CNN, object localization, feature maps, object detection.

\section{INTRODUCTION}

Because of the solid ability of highlight learning, CNN-based methodologies have accomplished the cutting-edge execution in various vision undertakings, for example, picture classification and article recognition. Nonetheless, the interpretability of CNNs is regularly condemned by the network, as these organizations normally look like confounded secret elements with monstrous unexplained boundaries. Subsequently, it is exceptionally attractive and important to find an approach to comprehend and clarify what precisely CNNs realized particularly for applications where interpretability is basic [1].

A significant issue in CNN learning is to clarify why classification CNNs anticipate what they foresee. Since both semantic and spatial data can be protected in element guides of profound layers, Gradient-weighted (Grad-CAM) was introduced to determine huge zones of data for CNN's gauge using significant component [2].

$\mathrm{CAM}++$ is one of the most notable implementation algorithms that performs well on once-over to verify everything is normal among best-in-class translation calculations as of late concentrated in. Thus, we decide to consider the rightness of Grad-CAM++. Additionally, we show that our outcomes even though tuned for Grad$\mathrm{CAM}++$, can move straightforwardly to Occluding Patch as another translation calculation. The area propositionbased framework (Mask RCNN), a two-venture measure, coordinates the attentional system of human cerebrum somewhat, which gives a coarse sweep of the entire situation right off the bat and afterward centres around locales of interest. The [3] the most delegate. Therefore, Mask R-CNN is easy for adaptable and productive structure for example level acknowledgment, which can be effectively summed up to different assignments with negligible change. 


\section{RELATED WORK}

In [4] presents a novel localization procedure. Additionally, we solidified our Grad-CAM constraints with existing significant standard insights to get significant standard class-discriminative Guided Grad-CAM portrayals. We accept that a genuine AI framework ought not exclusively be smart, yet in addition have the option to reason about its convictions and activities for people to confide in it.

The authors [5] introduced a method called (CAM) for distinguishing locales utilized by a confined class of picture characterization. Conversely, we make existing best in class profound models interpretable without modifying their engineering, accordingly, evading the interpretability versus exactness compromise. Our methodology is a speculation of CAM and is appropriate to an altogether more extensive scope of CNN model families: discriminative locales utilized by a limited class of picture grouping CNNs which don't contain any completely associated layers. make existing cutting-edge profound models interpretable without adjusting their engineering, subsequently.

The [7] examined the likelihood to encourage the CAM interpretability of an organization simply by alteration of the misfortune work. For this reason, we presented three GradCAM based interpretability quantifies and utilized the GradCAM entropy as extra misfortune term. An analysis using Resnet50, and PASCAL VOC information exhibited the practicality.

In [8] proposed new approach for understanding different layer of neural network and to identify task we use break down models for analysing application and identifying object. work, probabilistic methodology and anglebased methodology have been utilized with the end goal of article restriction. Mathematical mean of heatmaps of both the methodologies has been finished. In the previous methodology, the genuine article's slopes are made to stream into the last convolutional layer [6] to decide the main focuses which would assist with foreseeing that specific item. In the probabilistic methodology, CNN's top-down consideration has been utilized which fills the need of age of consideration maps which are task explicit.

The [9] have exhibited the preparation and testing of Mask-RCNN based individuals finders for top-see fisheye pictures. Indeed, even with the restricted assortment of commented on preparing information, we can fundamentally improve the location execution over the standard model. What is more, we have likewise researched two procedures appropriate for fisheye pictures and showed their capacities to additionally improve discovery results. Training with foundation pictures is additionally material for frameworks with fixed cameras (fisheye or point of view). In [10] This paper states the importance of Mask RCNN to have more unobtrusive model.

\section{PROPOSED METHOD}

The proposed algorithm to learn antagonistic patches that when stuck on the data picture, can change the interpretation of the model's conjecture. We will focus in on Grad-CAM++ in arranging our computations and a while later, show that our results summarize to other interpretation figurines too. Establishment on Grad-CAM++ portrayal Consider a significant association for picture gathering task. Negative pixels are likely going to have a spot with various characterizations in the image. Exactly as expected, without this ReLU, imprisonment maps every so often include something past the ideal class and perform all the more horrendous at restriction. Evacuation looks at are available in Sec. B.

we investigate the relationship among GradCAM and (CAM) [13], by officially displaying to determine the wide blend of CNN model, the Overview of CAM passes to identify the picture gathering CNN with a to determine the image using softmax. In particular, let the penultimate layer produce $K$ segment maps, Ak $\in R \mathrm{u} \times \mathrm{v}$, with each element by I, j. So Ak ij suggests the initiation at district $(I, j)$ of the part map Ak. To determine the GAP we use component maps are then spatially pooled and convey them to predict the value of Yc for each class $\mathrm{c}$

To get the Grad-CAM++ Lc Grad-CAM++ $\in \mathrm{R} u \times v$ of by determining the width $u$ and height $v$ for any class $c$, at first process we determine the value for class $\mathrm{c}$, yc with the value of the map Ak of a layer, (yc/Ak ). The width and stature estimations (requested by I and $\mathrm{j}$ independently) to get the neuron hugeness loads ack :

$$
\alpha_{k}^{c}=\overbrace{\frac{1}{Z} \sum_{i} \sum_{j}}^{\text {global average pooling }} \underbrace{\frac{\partial y^{c}}{\partial A_{i j}^{k}}}_{\text {gradients via backprop }}
$$




$$
L^{c}=\operatorname{ReLU} \underbrace{\left(\sum_{k} \alpha_{k}^{c} A^{k}\right)}_{\text {linear combination }}
$$

During Computation of $\alpha \mathrm{ck}$ while back inciting tendencies with respect to inceptions, the particular count amounts to reformist cross section aftereffects of the weight networks and the point concerning activation limits . Hereafter, the weight $\alpha$ ck determine the partial linearization.

The proportionality steady $(1 / \mathrm{Z})$ represent, the articulation for wck is indistinguishable from ack utilized by Grad-CAM++ . In this manner, Grad-CAM++ is an exacting speculation of Grad-CAM.

\section{MASK R-CNN}

It starts with the process of sorting and refining the anchors. A positive and a negative anchor is proposed around every object which is then refined further. These anchors can be customized according to the kind of objects one wants to detect. Furthermore, these anchors play a significant role in the overall performance of Mask R-CNN. This is followed by the appearance of bounding boxes/regions because of the region proposal network. This is a very crucial point because region proposal network decides whether a particular object is a background or not and likewise bounding boxes are marked. The objects in the frame are determined by a classifier and a regressor by region of interest pooling. After refining of bounding boxes, masks are generated and placed on their appropriate positions on the object. This is the main distinguishable attribute of Mask R-CNN.

\section{Backbone Network}

This is the first network which processes the image in Mask R-CNN. Generally, ResNet50 or ResNet101 convolutional neural network is used for feature extraction. Primary and rudimentary features are extracted by the initial layers of this network. Whereas high-level feature extraction is accomplished by proceeding layers. The objects of different scales are detected by the Feature Pyramid Network (FPN). Even though pyramid rendering is eschewed because of computational and memory demand, pyramid hierarchy is used to obtain feature pyramids without heavily compromising the efficiency. FPN allows access of features at higher as well as lower levels shown in Fig 1.

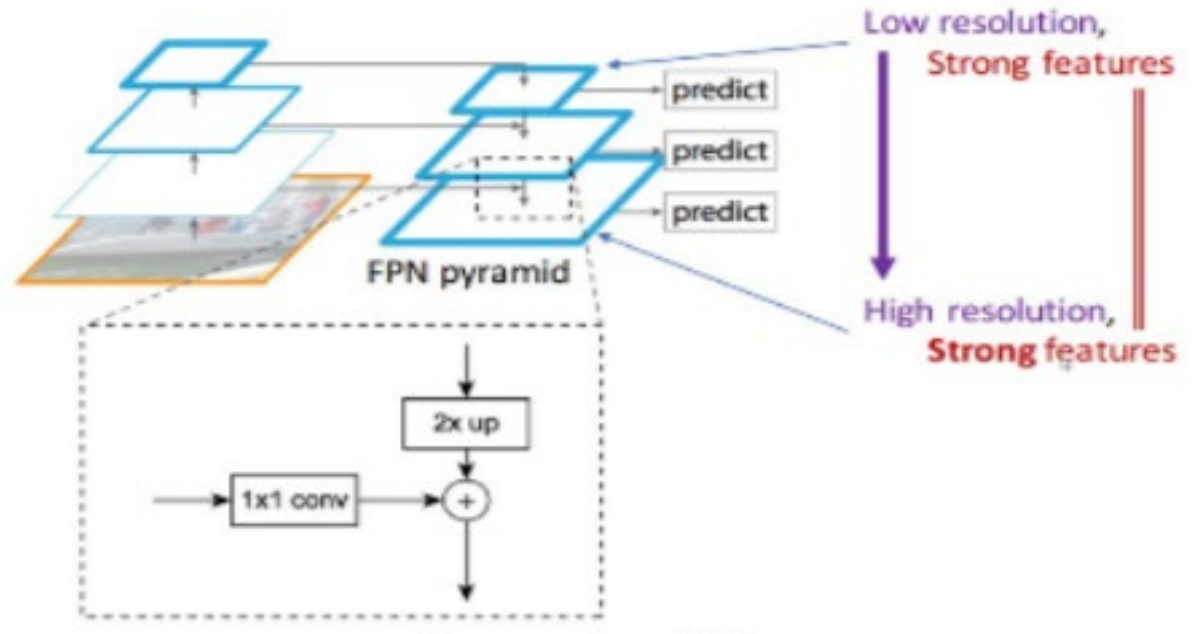

Representation of FPN

Fig 1. Backbone network of Mask RCNN

It can be termed as the backbone of Mask R-CNN. (RPN) is the first phase in Faster R-CNN. It is during this stage, the most suitable bounding boxes are determined along with the objectness score. These anchor boxes might vary in size because of the variance of object dimensions in the image. The classifier is trained in order to distinguish the background and foreground. Moreover, if the anchors are turned to vectors with two values and then fed to an 
activation function, labels can be predicted. A regressor of bounding box refines the anchors and a regressor loss function determines the regression loss.

\section{ROI Pooling and Classifier Training}

The RPN gives the most appropriate regions of bounding boxes. The predicament here is that they are of different dimensions. To eradicate this difference, ROI Pooling is applied in order to commensurate the feature maps of CNN.[11] In other words, ROI Pooling makes the process easier and efficient by converting many feature maps of different sizes into the same dimensions which makes a feasible structure for further processing. The feature map is divided into a fixed number of equal sized regions and then max pooling is applied to them. This makes the output of ROI Pooling constant irrespective of the size of the input. In this stage, features are extracted and can also be used for further conjectures. After this, RPN, classifier, and regressor can be trained altogether as well as distinctively. When the aforementioned three are trained together, the speed spikes up to $150 \%$ while the accuracy remains unhindered. Stochastic gradient descent and back-propagation can be used to train the region proposal network by following the image-centric sampling.

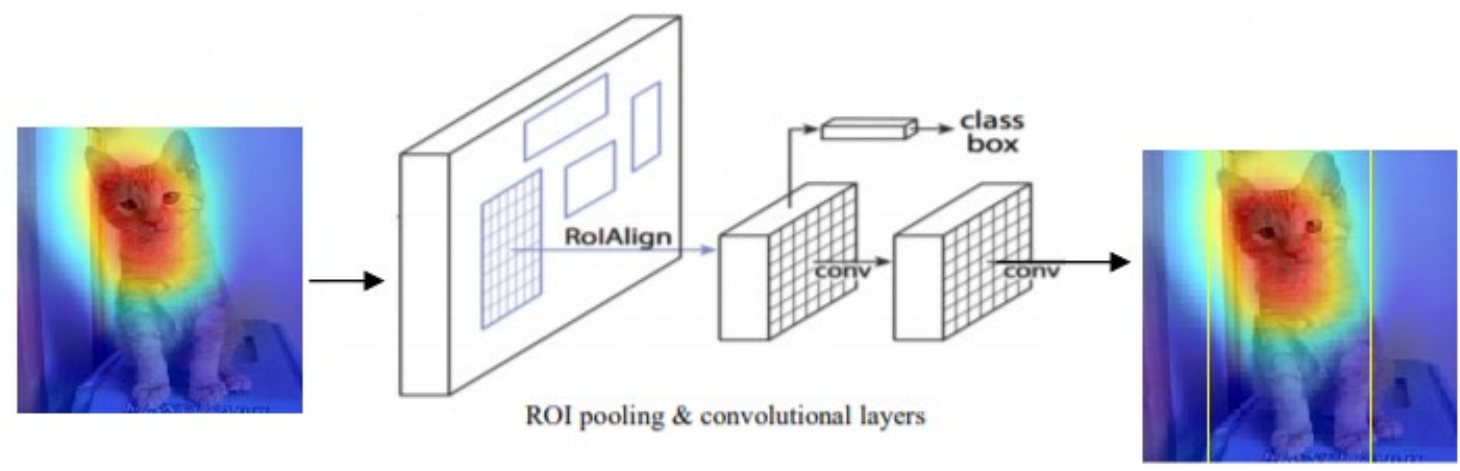

Fig 2. Mask RCNN output for object detection

Mask Generation and Layer Activation Mask is generated upon objects simultaneously while bounding boxes are predicted by the RPN. The process of instance segmentation is the key feature behind generating the masks for the objects in an image. This occurs at the pixel level and is the extension of Faster R-CNN which results in Mask RCNN. Low-intensity masks are generated in accordance with the classifier. These soft masks are more meticulous than binary masks. The loss is calculated during training and then the object mask is augmented to the size of bounding boxes. Moreover, activations of layers can be examined individually for detecting the noises (if any) and to rectify it. This generates a mask for each object in accordance with the input.

\section{RESULTS AND DISCUSSION}

The usage is finished utilizing MATLAB 2020a and utilizes ResNet101 as the spine organization. Because of the generally little size of the dataset, we utilize a Mask R-CNN model pre-prepared on the MS-COCO dataset as our beginning point. Prior to preparing we at first resize the pictures to $256 \times 256$ pixels. To ease the prerequisite of Mask RCNN for bigger preparing information. We explored different avenues regarding the learning pace of the model in 0.00001, While preparing the organization we began preparing the underlying layers in initial two ages and afterward prepared the entire Mask R-CNN start to finish alongside learning rate tempering. After testing a few gatherings of boundary mixes experimentally, we get the best outcome utilizing learning rate tempering by beginning with 0.00001 . At long last we perform post handling by eliminating location aftereffects of a similar class with more than 0.85 Intersection Over-Union among jumping boxes. In these cases, the jumping box with the bigger region was held while the more modest bouncing box disposed of from our location results.

The bouncing boxes were chosen whose class name coordinated with the ground truth and afterward those with a more noteworthy than half Intersection-Over-Union were picked. At last, the Accuracy for these classes was determined and plotted in Fig 3. and Fig 4 shows that the Grad-CAM++ representations for 'tiger feline' and 'fighter (feline)' separately. Fig 3 shows that the info picture given to handle the Grad-CAM++ and the recognized feline utilizing MASK RCNN alongside Grad-CAM++. 
TABLE 1. Iaccuracy Chart

\begin{tabular}{lll}
\hline Algorithm & $\begin{array}{l}\text { Training } \\
\text { accuracy }\end{array}$ & $\begin{array}{l}\text { Testing } \\
\text { accuracy }\end{array}$ \\
\hline CNN & 76 & 79 \\
MASK- & 80 & 81 \\
$\begin{array}{l}\text { RCNN } \\
\text { GRAD-CAM }\end{array}$ & 80 & 87 \\
MASK- & 82 & 90 \\
RCNN and & & \\
GRAD-CAM & & \\
\hline
\end{tabular}

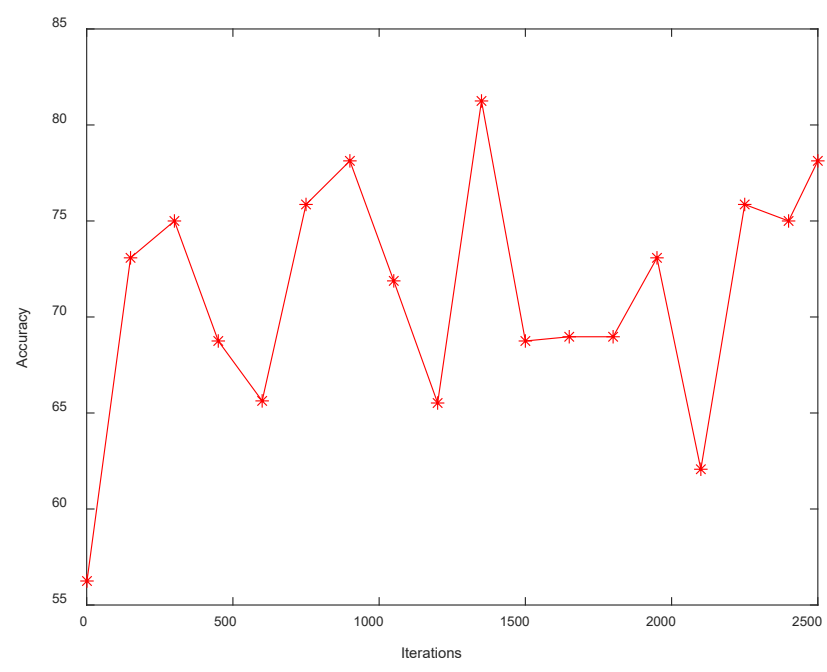

Fig 3. Result of Prediction accuracy

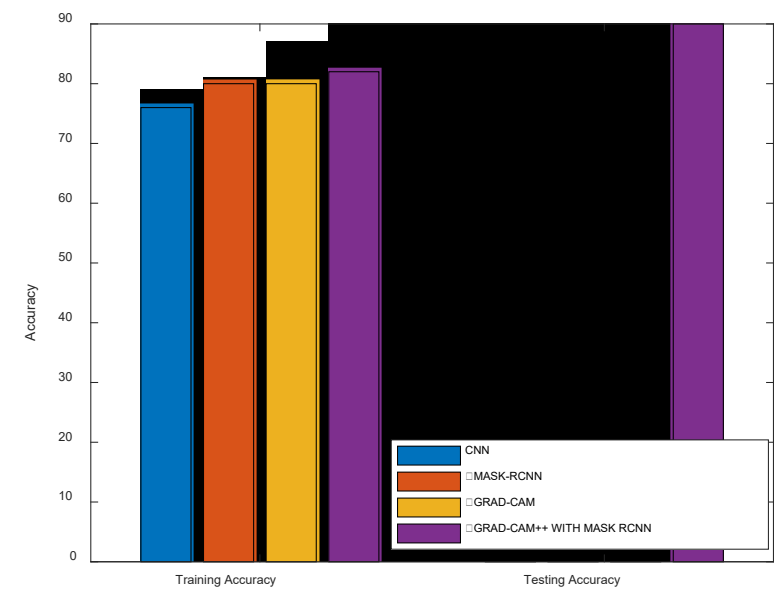

Fig 4. Accuracy comparison with existing methods 
From Fig 5, we can see that our proposed algorithm gives prediction accuracy comparing with the existing methods.
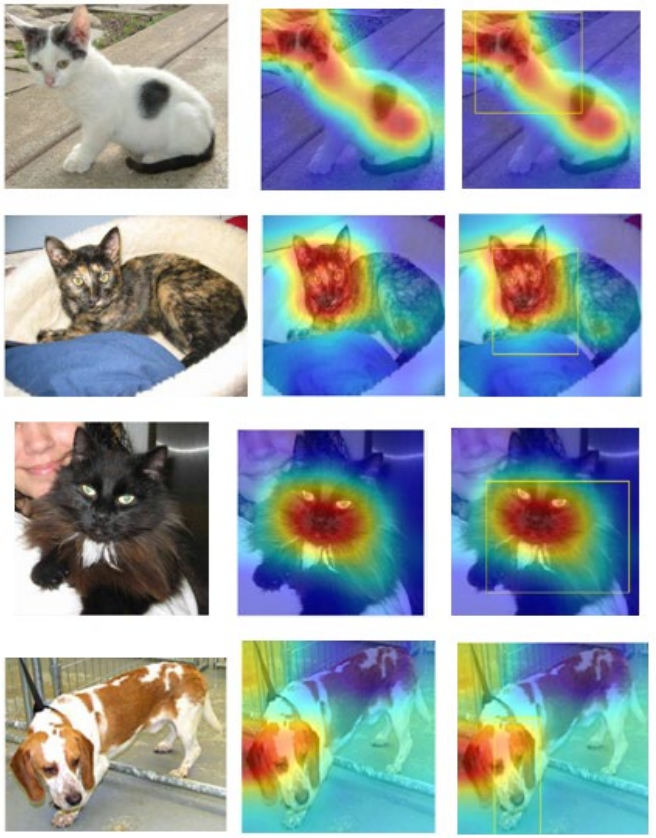

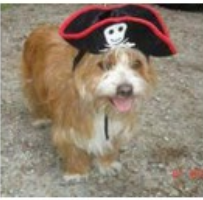

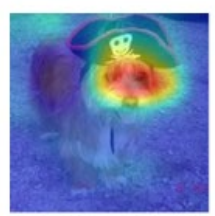

b

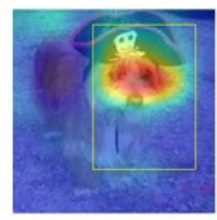

$c$

Fig 5. Results of proposed method

\section{CONCLUSION}

Thus, we have proposed a new technique for Grad-CAM++, to evaluate the CNN-based model more accurate by passing on visual clarifications. On the other hand, we have included Grad-CAM++ with MASK RCNN impediments with existing critical standard insight frameworks to pick up the best huge norm and classdiscriminative Grad-CAM depictions. Our perceptions outsmart existing approaches on the two axes interpretability and devotion to momentous model. Wide human assessments uncover larger observations will segregate classes significantly more unquestionably, reveal the dependability of a classifier. Grad-CAM++ and give an approach to manage secure printed clarifications for model choices. we recognize that an ensured AI structure ought not exclusively be quick, yet besides have the decision to response about its emotions and to build trust among people activities it combines the choices done during critical relationship in spaces, for example, maintain learning, normal language managing and video applications

\section{References}

[1]. M. Ilyas, A. K. Lakshmanan, A. V. Le, and M. R. Elara, "Staircase Recognition and Localization Using Convolution Neural Network (CNN) for Cleaning Robot Application," Dec. 2018.

[2]. H. Nonaka, "An ultrasonic 3-D object identification system using pulse neural network," AIP Conference Proceedings, 2000.

[3]. M. Agarwal, S. K. Gupta, and K. K. Biswas, "Grape Disease Identification Using Convolution Neural Network," 2019 23rd International Computer Science and Engineering Conference (ICSEC), Oct. 2019.

[4]. S. Kavitha, "Malarial Parasite Identification Using Convolution Neural Network," Bioscience Biotechnology Research Communications, vol. 13, no. 11, pp. 52-54, Dec. 2020.

[5]. H. Potlabathini, "Convolution Neural Network for Cooking State Recognition using VGG19," State Recognition symposium, Apr. 2019.

[6]. P. Jhinkwan, V. Ingale, and S. Chaturvedi, "Object Detection Using Convolution Neural Networks," SSRN Electronic Journal, 2019. 
[7]. K. Park and D. J. Cannon, "Recognition and localization of a 3D polyhedral object using a neural network," Proceedings of IEEE International Conference on Robotics and Automation.

[8]. E. Etemad and Q. Gao, "Object localization by optimizing convolutional neural network detection score using generic edge features," 2017 IEEE International Conference on Image Processing (ICIP), Sep. 2017.

[9]. S. Baskaran, J. Korrapati, S. K., and P. R., "Identification of Violence Images Using Convolution Neural Network in Social Network," International Innovative Research Journal of Engineering and Technology, vol. 5, no. 3, pp. 34-38, Mar. 2020.

[10]. J. Krejsa and S. Věchet, "Utilization Of Convolution Neural Network Based Road Detection In Mobile Robot Localization," Engineering Mechanics 2019, May 2019.

[11]. F. Mustapha, S. Sapuan, K. Worden, and G. Manson, "Damage Identification and Localization of Carbon Fiber-Reinforced Plastic Composite Plate Using Outlier Analysis and Multilayer Perceptron Neural Network," Composite Materials Technology, pp. 79-113, Dec. 2009.

[12]. E. Dong, Y. Zhu, Y. Ji, and S. Du, “An Improved Convolution Neural Network for Object Detection Using YOLOv2,” 2018 IEEE International Conference on Mechatronics and Automation (ICMA), Aug. 2018. 